\title{
Link Aware and Energy Efficient Routing Algorithm in Wireless Body Area Networks
}

\author{
Hajar Khodabandeh $^{1}$, Vahid Ayatollahitafti ${ }^{2,}$, Mohammad Sadeq Taghizadeh $^{3}$ \\ ${ }^{1}$ Department of Computer Science, Yazd Branch, Islamic Azad University, Yazd, Iran \\ E-mail: khodabandehhajar@gmail.com \\ 2,* Department of Computer Science, Taft Branch, Islamic Azad University, Taft, Yazd, \\ Iran \\ E-mail: vahid.ayat@taftiau.ac.ir \\ ${ }^{3}$ Department of Computer Science, Mehriz Branch, Islamic Azad University, Yazd, Iran \\ E-mail: mohammad.sadegh.t@gmail.com
}

Received: April 14, 2017

DOI: 10.5296/npa.v9i1-2.11547
Accepted: June 30, 2017

Published: June 30, 2017

URL: https://doi.org/10.5296/npa.v9i1-2.11547

\begin{abstract}
Due to increasing developments of medical science, early detection and receiving exact information in treatment of diseases and preventing them are very important. Body Area Networks (BANs), a subset of Wireless Sensor Networks (WSNs), deliver vital signs of patients to physician by collecting data applying different types of medical sensors. One of the most significant challenges faced, is the design of a link aware routing algorithm with different data priority. In this paper, a new link aware and energy efficient routing algorithm is proposed to select the efficient next hop node for the packets. Next hop node selection is based on the type of the packets. Results of simulation show that the proposed algorithm improves energy consumption, path loss and throughput compared to the similar routing algorithm.
\end{abstract}

Keywords: Routing, Wireless Body Sensor Networks, Link aware, Energy efficient 


\section{Introduction}

Achievements and new technologies in the field of electronic and wireless communication have empowered the development of the sensors with low energy consumption, small size, and reasonable price. Wireless Sensor Networks (WSN) are used in different applications such as security systems, military systems, monitoring systems, traffic control, medical care, and environmental control [1]. Body Area Networks (BANs) as special type of WSNs are applied in different fields especially in medical science for monitoring of the patient's body. Traditional health monitoring system copes with the high cost of healthcare. Small and smart sensors are attached to the human body as a result of the development of microelectronic and microelectromechanical systems. These devices collect vital signs of patient and send them to medical personnel such as pharmacists and nurses for more experiments and analysis [2,3].

The main idea behind BANs is to monitor patient's body on the hospital environment, and the working area of the remote location. Many improvements in technology give us the opportunity of merging all systems into one chip with the lowest cost. Another aspect discussed in BANs is merging the network with technologies, such as mobile phone, PDA and so on, using more attractive aspects such as fast delivery and a secure transformation of different information. BANs with fewer sensors may implant sensors in the body. Depending on the required parameters, different sensors and different network topology are essential. Another important point is that the wireless network should provide a proper action for a long time without battery recharging or replacing, especially in the body. Therefore, it is necessary to examine a well-designed network for its energy waste. Most of the essential aspects of the system's energy consumption include the main source of energy, continuous transmission of data, single hop communication, and distance between the nodes. In the case of communication, nodes that are far from the sink are less likely to be depleted, while in the multi-hop communication manner, the energy consumption of the nodes which are closer to the sink is higher than other nodes. Another challenge in this regard is the fact that in designing, routing protocols should consider the energy consumption and link utilization [4].

A number of energy efficient protocols [5-8] have been proposed for BANs and health monitoring which consider mobility of the body. However, the protocols do not consider priority of data generated by the sensor nodes on the body. Authors in [9] use priority of data but do not take into consideration path loss and link utilization. In this paper, a link aware and energy efficient routing algorithm has been proposed for BANs. In this algorithm, packets are classified into two categories: high priority and low priority packets. For the high priority packets, next hop node selection is based on the minimum hop counts and delay of the neighboring nodes to the sink. Furthermore, next hop selection for the low priority packets is based on two parameters: distance to the sink and path loss of the neighboring nodes.

Furthermore, we point out a brief review of related works on routing protocols in Section 2 and in Section 3, we discuss our proposed approach. Section 4 shows details of our simulations and evaluates the performance of the proposed approach. Lastly, Section 5 reveals the conclusion. 


\section{Related Works}

A number of studies have been conducted in the field of sensor networks with the aim of improving the performance of these systems. Nabi et al. [5] provided a robust protocol for wireless networks which is capable of transmitting power with high conformity. They proposed a multi hop protocol to monitor the health of the human body. The proposed protocol is resistant against frequent changes in network topology due to changes in the status of the wireless link quality and its diversity. Techniques for implementing transmission of sensor nodes at runtime make it possible to optimize power consumption to ensure the reliability of outputs for each node in the network. Nabi et al. [6] provided a mobility model that could be adjusted to BAN. In their action, a set of model was defined, as mobility model to evaluate the relationship of super BANs. Implementation of state position and active nodes on a particular situation in the model can be used on a wide variety of applications.

In another study, braem et al. [7] analyzed the requirements of body support mobility in a wireless network. This analysis was conducted in two categories: support of mobility, in particular the independence of locations, and increasing the resilience of the suspension time. The simulation results showed that the network in both cases had to enrich and motivate the location independence. Javaid et al. [10] provided energy-efficient model with new routing protocol for BAN. A new routing protocol in wireless sensor networks implemented for a heterogeneous body, which thermal threshold-based adaptive mobility took place with M-attempt protocol. Sandhu et al. [11] provided a mobile model in BAN and presented a mathematical model in order to stimulate nodes due to changing state. Yousaf et al. [4] introduced CEMob and emergency transfer of data to support mobility in BANs. Their work in this area reflects the fact that the proposed protocol CEMob, used as a routing layer protocols, to support network critical data transfers to emergency services with mobility in BANs. CEMob also prevent ongoing transmission of information, conserving energy in nodes. Ovesh et al. [12] investigated LAEEBA routing protocol in BANs. They changed LAEEBA (Link Aware and Energy Efficient protocol for wireless Body Area networks) protocol to enhance the performance of the protocol. Eight sensor nodes with similar capabilities and computing power deployed in the human body, and the sink node placed in the waist. Different nodes were used to measure various activities such as glucose.

Birari et al. [13] provided interference-free and reliable communication models with wireless mobility model in BAN's. Wireless mobility management in the mobile wireless network systems monitors the patient's body. In this model, a high level of electromagnetic radiation interferes with device's performance, mobility, wireless and reliable communication method is adopted for the patient. Vallejo et al. [14] provided instruction in the control of active and reactive power in order to save their physical energy in wireless sensor network. Ahmed et al. [15] evaluated the performance of BANs with Co-LAEEBA (Cooperative Link Aware and Energy Efficient protocol for wireless Body Area networks) protocol. Smail et al. [16] introduced energy-aware routing protocol for wireless networks with sustained structure. In This protocol they used a model with energy-efficient road link. The simulation results show that in the protocol, the upper limit energy consumption was improved. Kavita et al. [17] provided a transmission path in terms of energy efficiency programs for BAN. 


\section{Proposed approach}

The proposed approach is the improvement of Co-LAEEBA's protocol. In this Protocol, a path with the lowest hop counts is chosen. In the proposed approach, the multi-hop path is used for data transferring. Relay nodes can easily transfer incoming data to the sink just with their participation. Co-LEEBA is a protocol on the principles of partnership, in which the number of nodes act as coordinating nodes. The ordinary nodes are allowed to send their information in each round. Input and output data except the source node must be equal to each peer node. A path between peer nodes and sink has a high data transmission capacity.

In the proposed approach, the packet is divided into two groups: high and low priority packets. For high priority packets, node which has the lowest hop counts to the sink and the least delay to the next node is chosen. For low priority packets, node which is closer to the sink and has the lowest path loss is chosen.

\subsection{Network topology}

In the network, the sink is located on the human body. The network is heterogeneous, which means that there are two types of sensors: sensors with high priority and sensors with low priority. Figure 1 shows the position of sensors in the human body related to the $\mathrm{X}$ and $\mathrm{Y}$ coordination. The blue sensors represent the normal sensors while the red one represents the sink node.

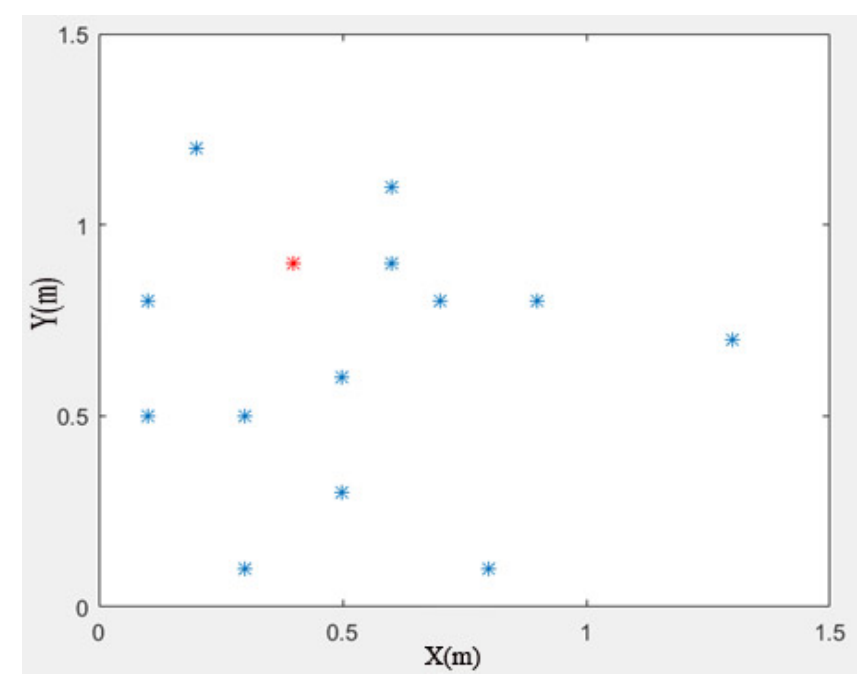

Figure 1. Position of sensors in the human body

\subsection{Initialization}

In the initialization phase, the assumptions are as follows:

- Each node is aware of the position of its neighboring nodes.

- Nodes are well aware of their situations.

- All possible routes as well assessed. 


\section{Macrothink}

- In each node, a packet that includes ID information, location and initial energy is transmitted.

After network configuration and parameter setting, routing and relations between cooperative nodes are determined. After identifying the possible routes, function optimization is performed.

\subsection{Routing and participation}

In order to describe the routing phase, a pair of origin-destination node is considered to be separated by a space. All stages of work are done in two phases shown in Figure 2. In the first phase, the source $\mathrm{S}$ simultaneously sends data to the relay node $\mathrm{R}$ and destination $\mathrm{D}$. In the second phase, node $\mathrm{R}$ receives information transmitted from $\mathrm{S}$ to $\mathrm{D}$. The distance between the relay node and the source node is $\mathrm{d} 1$ and the distance between the relay node and the destination is equal to $\mathrm{d} 2$.

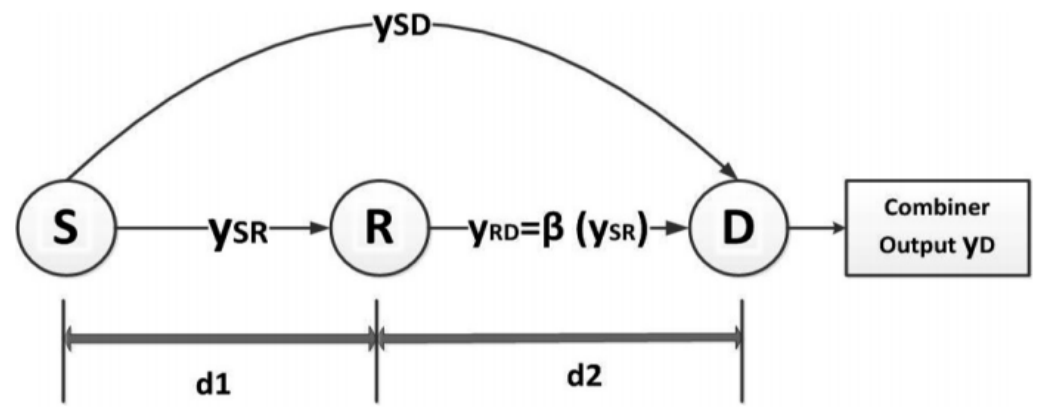

Figure 2. Linear three-sensor system model

Information received from the node $\mathrm{R}$ and $\mathrm{D}$ of origin, in the first phase, can be calculated as follows:

$$
\begin{gathered}
Y_{S R}=h_{S R} x_{S}+N_{S R} \\
y_{S D}=h_{S D} x_{S}+N_{S D}
\end{gathered}
$$

where $\mathrm{X}_{\mathrm{S}}$ is the transmitted data symbol from $\mathrm{S}, \mathrm{h}_{\mathrm{SR}}$ and $\mathrm{h}_{\mathrm{SD}}$ are the wireless medium characteristics from $\mathrm{S}$ to $\mathrm{R}$ and $\mathrm{S}$ to $\mathrm{D}$. These parameters are modeled as a random variable with Gaussian distribution. $\mathrm{N}_{\mathrm{SR}}$ and $\mathrm{N}_{\mathrm{SD}}$ are noise nodes introduced between nodes of $\mathrm{S}$ to $\mathrm{R}$ and $\mathrm{S}$ to $\mathrm{D}$. In the second phase, $\mathrm{R}$ transmits incoming signal from $\mathrm{S}$ to $\mathrm{D}$, after processing on it.

\subsection{The route selection}

To reduce and to balance the energy consumption, peer nodes are chosen as the sender node. Sink node has all information about ID, distance and remaining energy of all nodes. These nodes calculate the cost function of all nodes and then decide which node is a perfect 
choice as cooperative nodes and which nodes are able to send your data directly to the sink. Cost function of nodes can be calculated as follows. If $\mathrm{E}_{\mathrm{re}}(\mathrm{S})>\mathrm{E}_{\mathrm{re}}(\mathrm{R})$, communication is in direct communication, else communication is based on multi-hop manner.

In order to maximize the remaining energy and if there is more than one $\mathrm{R}$ node for $\mathrm{S}$, then $\mathrm{R}$ nodes with more $\mathrm{E}_{\mathrm{re}}$ and shorter distance from $\mathrm{S}$ node are chosen. Nodes with this condition are chosen as peer nodes.

\subsection{Energy}

The power consumption in a single hop, calculated as follows:

$$
E_{S-H O P}=\left(P_{\text {amp }, S D}+P_{S}+P_{D}\right) / R_{b}
$$

where $\mathrm{P}_{\mathrm{amp}}$, SD is the power consumed by the transmit amplifier, which is used for the peak-to-average rate modulation scheme and the accuracy of the transmission. $\mathrm{P}_{\mathrm{S}}$ and $\mathrm{P}_{\mathrm{D}}$ is power consumption for transmitting and receiving. $R_{b}$ is a data transfer rate, measured in bits per second. In a multi hop connection, the total energy consumption can be calculated as follows:

$$
E_{M-H O P}=\left(P_{a m p, S D}+P_{s}+2 P_{R}\right) / R_{b}+\left(P_{a m p, S D}+P_{s}+2 P_{D}\right) / R_{D}
$$

where the first term corresponds to the transmission from $\mathrm{S}$ to $\mathrm{R}$, and the second term corresponds to the transmission from $\mathrm{S}$ to $\mathrm{D}$.

\subsection{Packet Prioritization}

In the proposed approach, data packets are categorized into two categories: high-priority and low-priority packets. High priority packets should transfer data to the sink faster. Thus, for high priority packets, nodes are chosen as the next hop, which have the lowest number of node interface to the sink and the least delay to the next nodes. For the low-priority packets, nodes, which are closer to the sink and their path loss are minimum, are chosen as the next hop nodes. The assumption is made that each node is aware of the number of neighboring nodes as well as the delay and loss of the path.

\subsection{Selecting the next hop node}

Selecting the next hop is based on the priority of the packets. The way of choosing the next hop node for low-priority packets is presented in algorithm 1. NH has a set of neighbor nodes with the lowest number of hop counts to the sink (line 2). If the value of NH is null (line 3), the next hop is empty (line 4). In this situation, there is no neighboring node to send information. If NH has one member (line 5), that member is elected as the next hop (line 6). If there is more than one member NH (line 7), the nodes are selected for the next hop with the minimum path loss (line 8). 


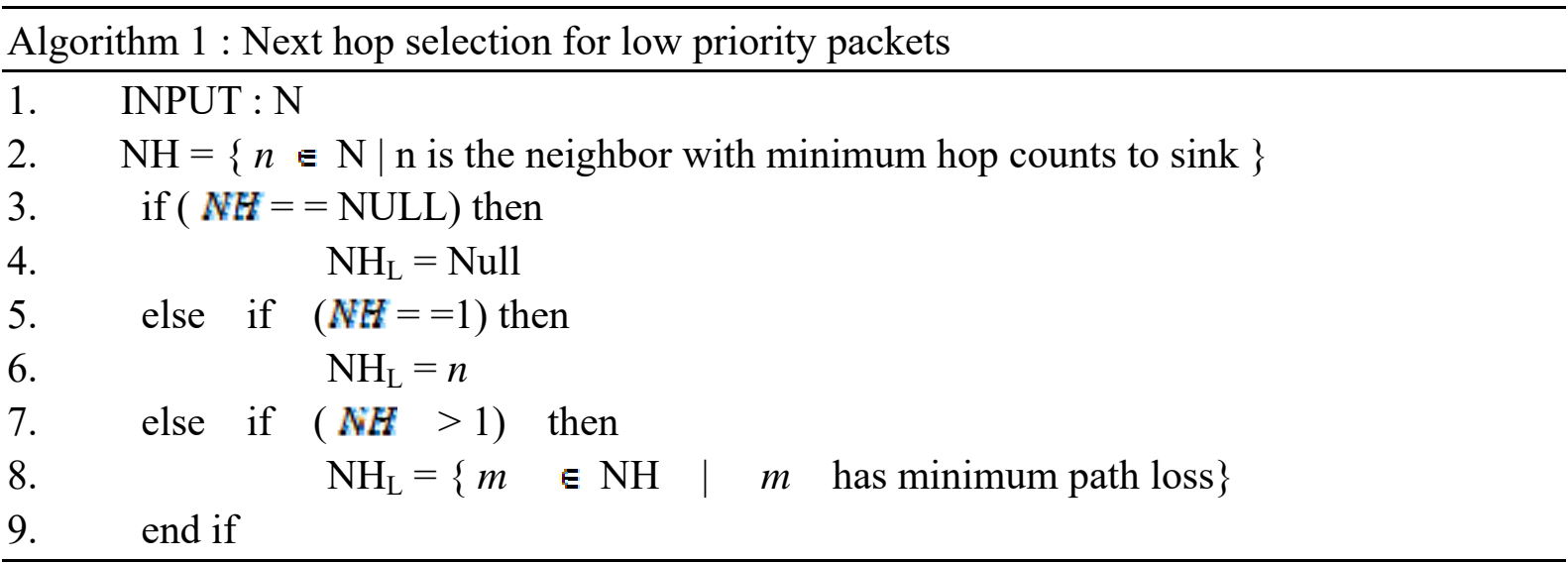

Next hop node selection for the packets with high priority is shown in algorithm 2 . The set of NH is the neighboring nodes with the lowest hop counts (line 2). If the value of NH is null (line 3 ), the next hop is empty (line 4). This condition occurs when no neighboring node exist for sending information. If the set NH has one member (line 5), that member is selected for the next hop (line 6). If there is more than one member in NH (line 7), the members with the lowest delay to the neighboring nodes are selected as the next hop node (line 8).

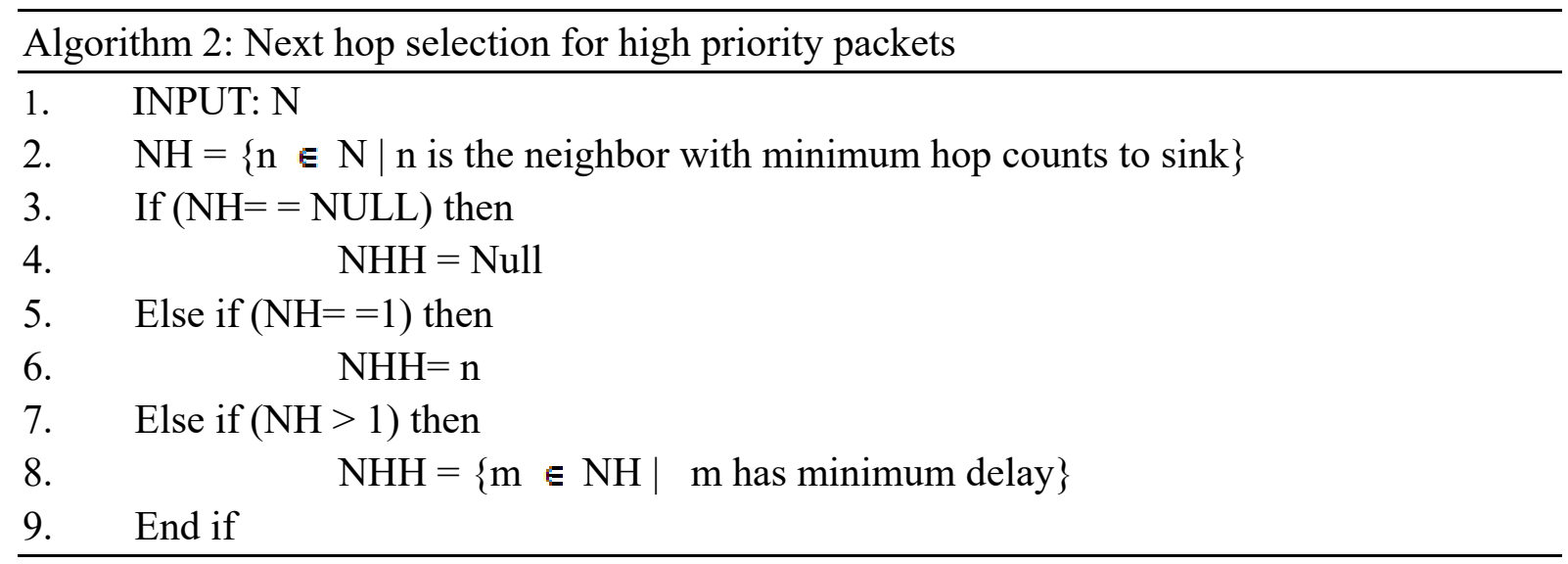

\section{Performance Evaluation}

The improvement of Co-LEEBA is implemented and evaluated in this section. The most important difference in the proposed approach and Co-LEEBA is the division of packets into two groups with high and low priority. For packets with high priority, the node with the lowest delay and the lowest hop counts to the sink is selected. For packets with lower priority, node selection is based on the lowest distance from the sink and the lowest path loss. In order to evaluate the proposed approach, energy consumption, path loss and throughput diagrams are calculated and the results are compared with Co-LEEBA.

Figure 3 shows the final result obtained by applying Co-LEEBA to the network. In this figure, the green lines represent the routes between the nodes and red lines represent direct routes from the nodes to the sink. 


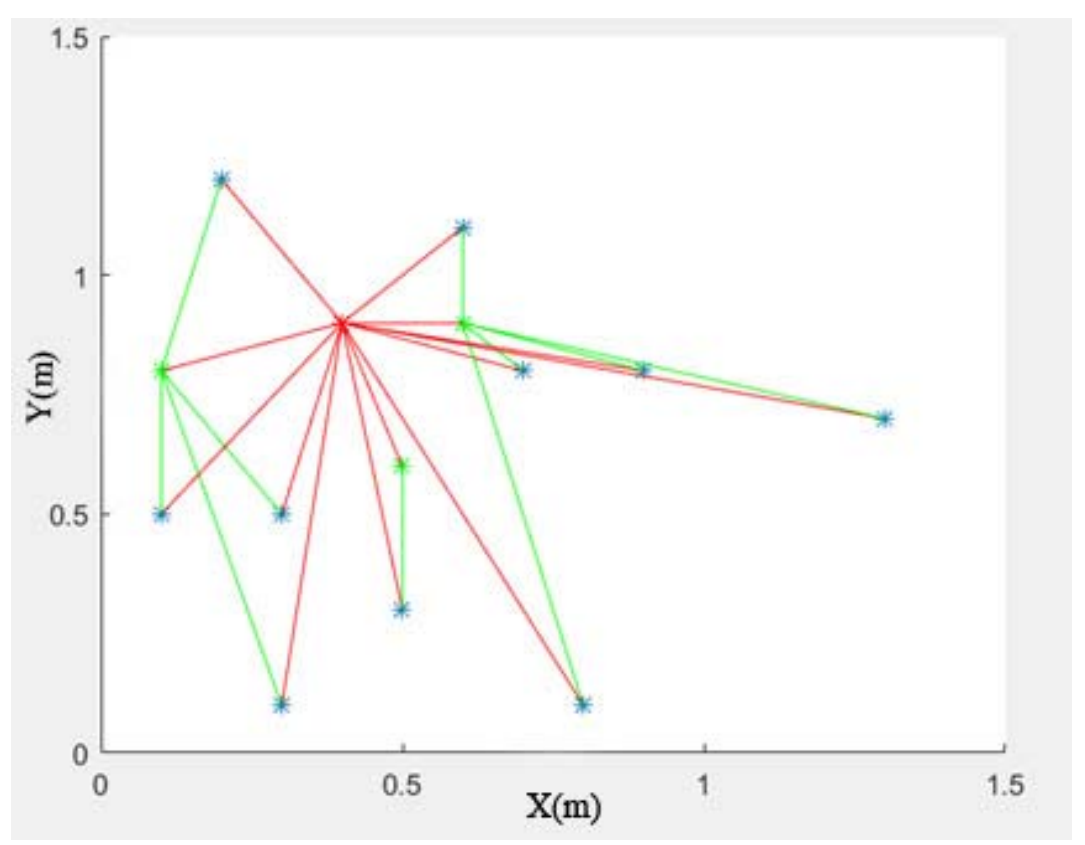

Figure 3. The final result of routing in the C0-LEEBA algorithm

We use MATLAB (2016) simulator to simulate the proposed approach. The most important parameters used for simulation are presented in table 1 . The initial energy of the sink is supposed to be unlimited.

Table 1. Simulation parameters

\begin{tabular}{|c|c|}
\hline Parameter & Value \\
\hline $\mathrm{E}_{\mathrm{rx}}$ & $36.1 \mathrm{~nJ} / \mathrm{bit}$ \\
\hline $\mathrm{E}_{\mathrm{tx}}$ & $16.7 \mathrm{~nJ} / \mathrm{bit}$ \\
\hline $\mathrm{E}_{\mathrm{amp}}$ & $1.97 \mathrm{pJ} / \mathrm{bit}$ \\
\hline $\mathrm{E}_{\mathrm{DA}}$ & $5 \mathrm{~nJ} / \mathrm{bit}$ \\
\hline Freq. & $2.4 \mathrm{Ghz}$ \\
\hline Initial energy & $0.6 \mathrm{~J}$ \\
\hline Packet size & $1000 \mathrm{~b}$ \\
\hline
\end{tabular}

As mentioned, in the proposed approach, in addition to the assumptions which is considered in the C0-LEEBA algorithm, it is assumed that the packet has high and low priority. Figure 4 shows the results of the proposed approach and its performance on the network. In this figure, the red routes, belongs to the node containing low-priority packets. As it can be seen, in these nodes, packets have moved to the shortest distance from the sink. Yellow routes belong to the nodes that contain high-priority packets. It can be seen that the nodes have chosen other nodes as the next destination with the lowest number of hops to the sink and also has the lowest delay to the next node. 


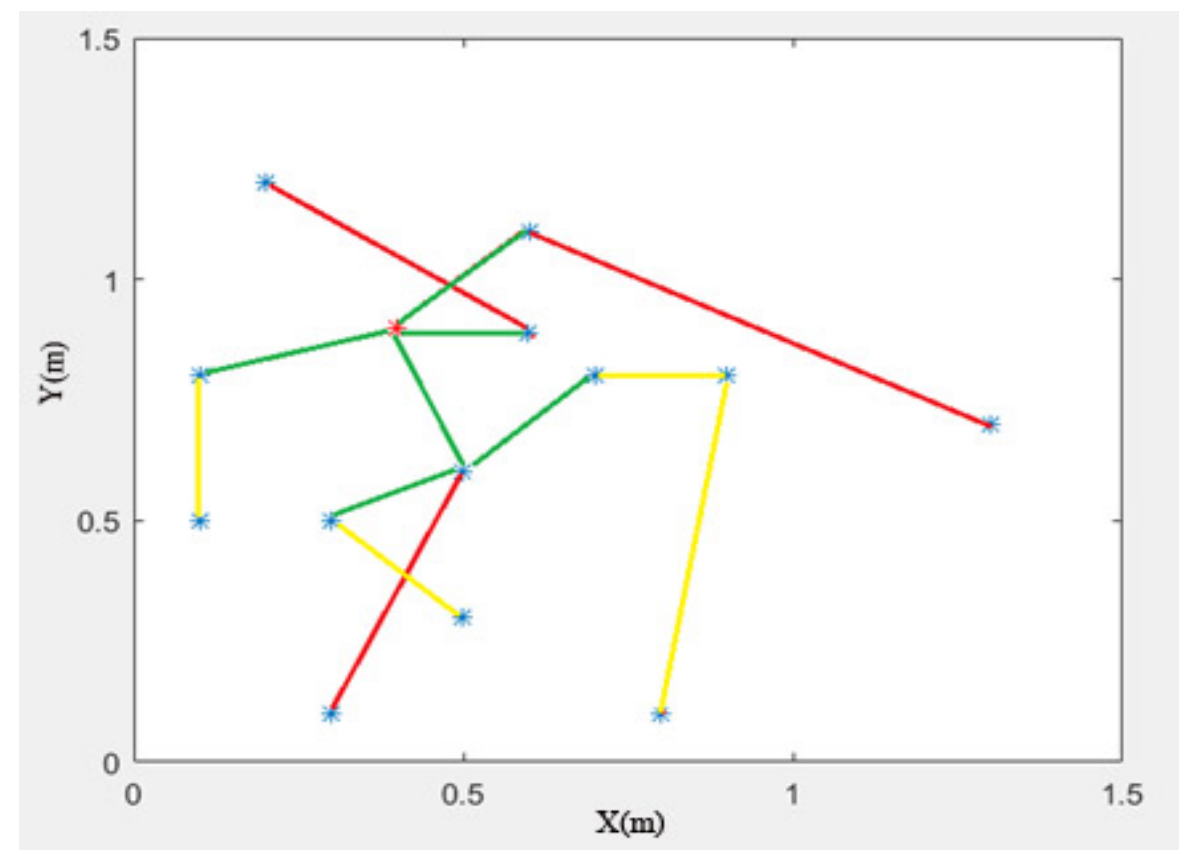

Figure 4. An example of routing in the proposed approach

In the proposed approach, there are two types of sensor with high and low priority. Figure 5 shows the residual energy of the network over time. At first, the residual energy of the whole network, which is equal to the total residual energy in sensors, is equal to 8 joules. During the network activity, this energy is gradually reduced. Figure 5 shows the remaining energy diagram in the network for two routing approaches. As it can be seen, the proposed approach saves system's energy over time, in greater amounts than Co-LEEBA could save. This is because in the proposed approach, minimum hop counts to the sink for next hop selection causes energy conservation. Furthermore, in time of 10000 s the nodes for Co-LEEBA and in time of 12000 s for the proposed approach, the nodes run out of energy.

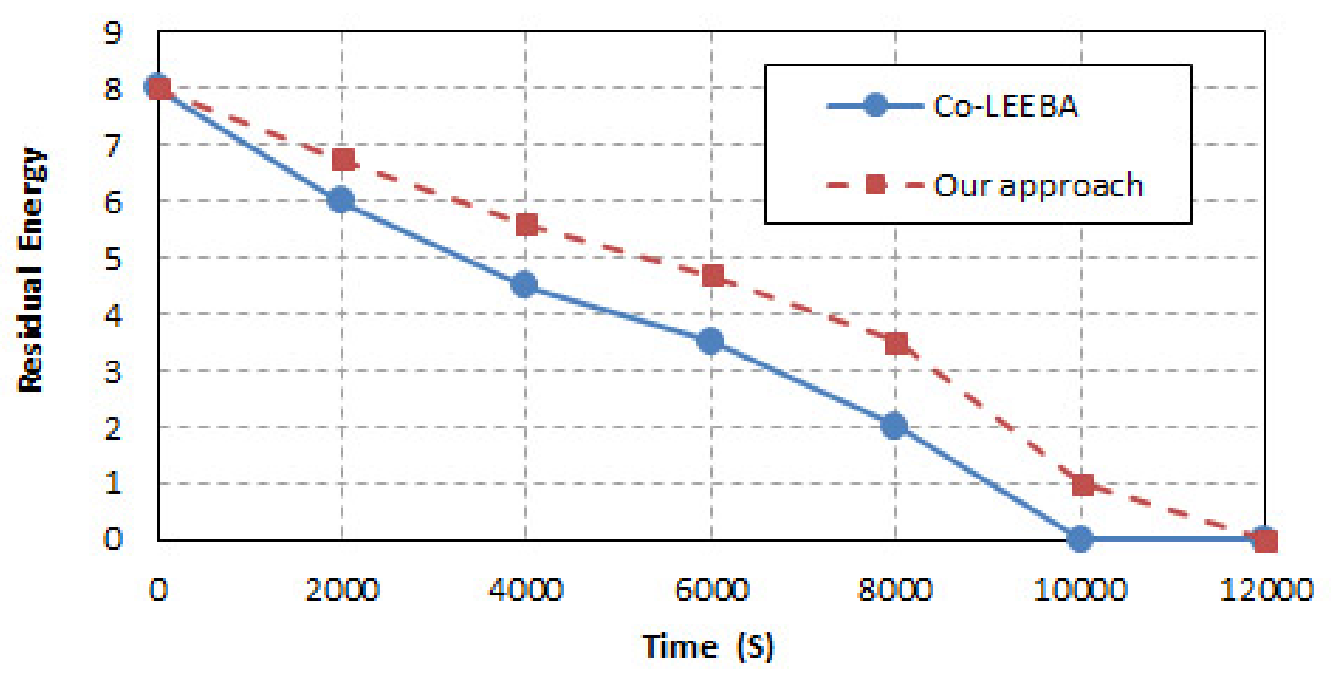

Figure 5. Residual energy(J) 
The energy consumption of the proposed approach and Co-LEEBA is shown in Figure 6. As it can be seen from the diagram, the proposed approach has the lower energy consumption related to the Co-LEEBA. This is because; in the proposed approach next hop selection is based on the minimum hop counts to the sink. Moreover, for high priority packets, next hop selection is based on the lowest path loss leading to the lower retransmitted packets and the lower energy consumption.

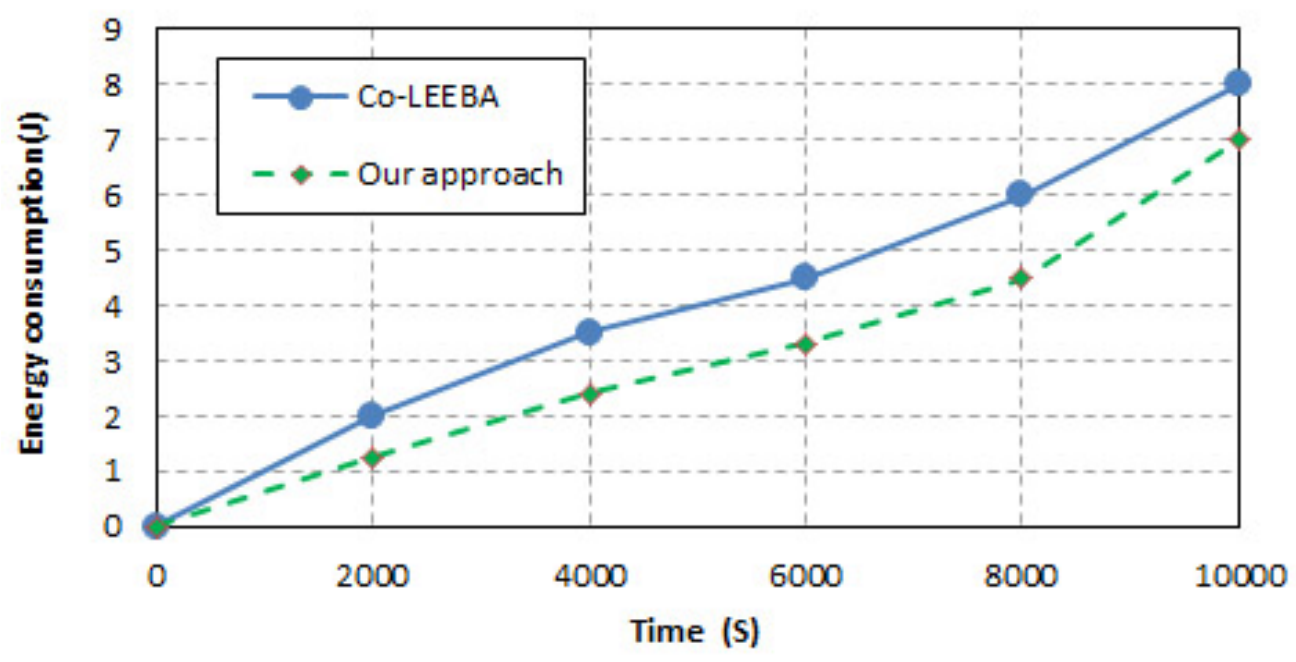

Figure 6. Energy consumption (J)

Path loss analysis presented in Figure 7 and the proposed approach compared with C0-LEEBA approach. As can be seen from the figure, the path loss of the proposed approach reaches to zero at time 5000s while in Co-LEEBA reaches to zero at time 8000s. Results show that the proposed approach, compared to Co-LEEBA approach, has the better performance in reducing path losses during the time. This path loss reduction is a result of considering nodes with minimum path loss for low priority routes.

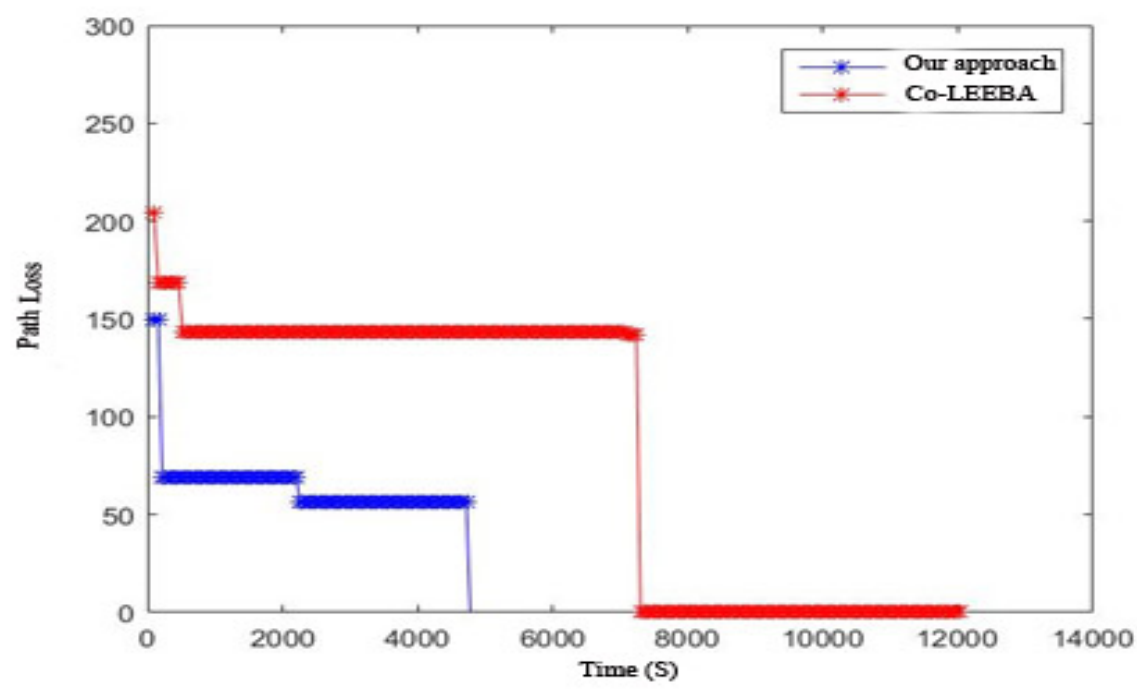

Figure 7. Path loss (dB) 
Throughput is defined as the number of packets that are successfully received in the sink. According to Figure 8, it is clear that the proposed approach has higher throughput than the base approach. The reason for this increasing is that in the proposed approach, the route with the minimum path loss is selected, which will result in more packets to reach the destination. Throughput of the proposed approach is about $\% 37$ lower than that of the Co-LEEBA approach.

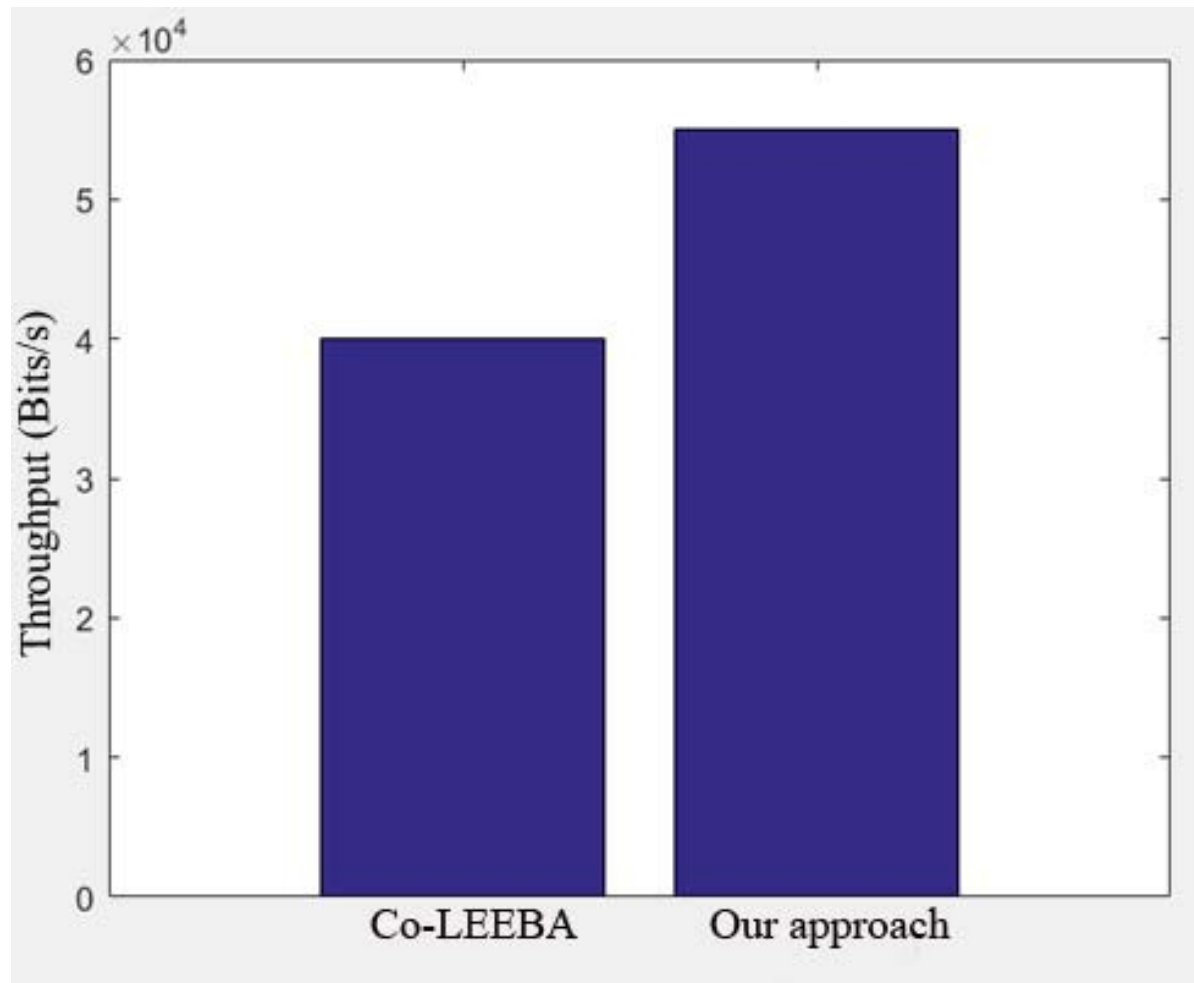

Figure 8. Throughput (Bits/s)

\section{Conclusion}

In this paper, packets were categorized into two priorities: high-priority and low-priority. High priority packets need to be forwarded to the sink faster. Therefore, they were designed in a way which the number of intermediate hops to the sink was minimized and had the lowest delay to the next node. For packets with the lower priority, the nearest node to the sink as well as the node with the lowest path loss was selected as the next hop node. The results presented in the simulations showed that the proposed approach has the lower energy consumption than Co-LEEBA because of the efficient next hop node selection. Furthermore, the proposed approach had lower path losses over time and greater throughput (about \%37 more than Co-LEEBA). In the future, we are interested in implementing Quality of Service requirements in WBANs. Additionally, thermal-aware routing can be integrated with link-aware routing. 


\section{References}

[1] Heidemann, J. and R, Govindan. "Embedded sensor networks". Handbook of Networked and Embedded Control Systems, 2005, Springer: 721-738. Available at http://www.springer.com/gp/book/9780817632397.

[2] Latré, B., Braem, B., Moerman, I., Blondia, B., and Demeester, P. “A survey on wireless body area networks". Wireless Networks. 17(1), 2011, pp. 1-18. https://doi.org/10.1007/s11276-010-0252-4

[3] Yousaf, S., Akbar M., Javaid, N., "Cemob: Critical data transmission in emergency with mobility support in wbans". 28th International Conference on Advanced Information Networking and Applications (AINA), IEEE, Victoria, BC, Canada, June 2014. https:// doi.org/10.1109/AINA.2014.111

[4] Lloret, J., Parra, L., Taha, M., Tomás, J., "An architecture and protocol for smart continuous eHealth monitoring using 5G." Computer Networks (2017). https://doi.org/10.1016/j.comnet.2017.05.018

[5] Nabi, N., Basten, T., Geilen, M., Blagojevic, M., Hendrics, T. "A robust protocol stack for multi-hop wireless body area networks with transmit power adaptation." Proceedings of the fifth international conference on body area networks. ACM, Corfu, Greece, September 2010 pp. 77-83. https:// doi.org/10.1145/2221924.2221941

[6] Nabi, M., M. Geilen, Basten, T., "MoBAN: A configurable mobility model for wireless body area networks". Proceedings of the 4th International ICST Conference on Simulation Tools and Techniques, ICST (Institute for Computer Sciences, Social-Informatics and Telecommunications Engineering), Barcelona, Spain, March 2011, pp. 168-177.

[7] Braem, B. and C. Blondia, "An analysis of requirements to supporting mobility in Body Area Networks". Computing, 2012 International Conference on Networking and Communications (ICNC), IEEE, Maui, HI, USA, Feb 2012. https://doi.org/10.1109/ICCNC.2012.6167555

[8] Atto, Muhsin, and Chris Guy. "Routing Protocols for Structural Health Monitoring of Bridges Using Wireless Sensor Networks." Network Protocols and Algorithms 7.1 (2015), pp. 1-23. https://doi.org/10.5296/npa.v7i1.7264

[9] Calhan, A. "A Non-Preemptive Priority Scheduling Algorithm for Improving Priority Data Transmission Delay in Wireless Body Area Networks." Adhoc \& Sensor Wireless Networks 34(1), 2016, pp. 59-75.

[10] Javaid, N., Abbas, Z., Fareed, M.S., Khan, Z.A., Alrajeh, M., "M-attempt: A new energy-efficient routing protocol for wireless body area sensor networks." Procedia Computer Science 19: 224-231. https://doi.org/10.1016/j.procs.2013.06.033

[11] Sandhu, M. M., Akbar, M., Behzad, M., "Mobility Model for WBANs". 2014 Ninth International Conference on Broadband and Wireless Computing, Communication and Applications (BWCCA), IEEE. Guangdong, China, Nov 2014. https://doi.org/10.1109/BWCCA.2014.60

[12] Ovesh, I. S. M. and Sharma A. K., "Modified LAEEBA Routing In WBAN." IJSRD-International Journal for Scientific Research \& Development,2014, 2(8), PP. 
2321-0613.

[13] Birari, V. M., Helonde, J., Vadhai, V., "Seamless Mobility with Interference Free Reliable Communication in WBAN". International Conference on Computing Communication Control and Automation (ICCUBEA), IEEE. Pune, India, Feb 2015. https://doi.org/10.1109/ICCUBEA.2015.54

[14] Vallejo, M., Recas, J., Ayala, L., "Proactive and Reactive Transmission Power Control for Energy-Efficient On-Body Communications". Sensors, 15(3): 5914-5934, 2015. https://doi.org/ 10.3390/s150305914

[15] Ahmad, A., Javaid, N., Qusim, U., Ishfaq, M., Khan, A., Alghamdi, T.A., "RE-ATTEMPT: a new energy-efficient routing protocol for wireless body area sensor networks". International Journal of Distributed Sensor Networks, 10.4, 2014. http://dx.doi.org/10.1155/2014/464010

[16] Smail, O., Kerrar, A., Zetili, Y., "ESR: Energy aware and Stable Routing protocol for WBAN networks". 12th International Wireless Communications \& Mobile Computing Conference (IWCMC 2016). Paphos, Cyprus, 2016. http://dx.doi.org/10.1109/IWCMC.2016.7577100

[17] Kavitha, D., Sundararajan, T., Hamsagayathri, P., Gunavathi, M. "ENERGY EFFICIENT TRANSMISSION PATH FOR WIRELESS BODY AREA NETWORK APPLICATIONS". International Research Journal of Engineering and Technology (IRJET), 3(4), 2016. Available at https://www.irjet.net/archives/V3/i4/IRJET-V3I493.pdf

\section{Copyright Disclaimer}

Copyright reserved by the author(s).

This article is an open-access article distributed under the terms and conditions of the Creative Commons Attribution license (http://creativecommons.org/licenses/by/3.0/). 